\title{
Editorial
}

Ingo Barkow*

\section{Das Schweizer Institut für Informationswissenschaft (SII)}

https://doi.org/10.1515/iwp-2020-2108

Liebe Leserinnen und Leser,

auf den nächsten Seiten werden Sie viel über die Studiengänge, die Forschungstätigkeiten und die Zukunft des Schweizer Instituts für Informationswissenschaft (SII) erfahren. Dafür nutzen wir gerne die Gelegenheit ein ganzes Schwerpunktheft der Zeitschrift Information - Wissenschaft und Praxis (IWP) mit unseren Inhalten zu gestalten und bedanken uns ganz herzlich bei der gesamten Redaktion für diese Möglichkeit. Für alle, die das Schweizer Institut für Informationswissenschaft (SII) und die Fachhochschule Graubünden (FHGR) nicht kennen, möchte ich an dieser Stelle kurz unsere Geschichte sowie unsere Schwerpunkte in Lehre und Forschung vorstellen.

Im Prinzip sind das SII als auch die FHGR alt und neu zugleich. Unsere Fachhochschule wurde zum 1. Januar 2020 als achte Fachhochschule der Schweiz aus der bisherigen Fachhochschule Ostschweiz (FHO) herausgelöst und dadurch autonom. Bis zu diesem Zeitpunkt waren wir als Hochschule für Technik und Wirtschaft (HTW) Chur eine Teilschule der Gesamthochschule. Für uns bedeutet die Autonomie eine deutliche Zäsur und eröffnet uns neue Möglichkeiten in der Studiengangsentwicklung, der Forschung und Themenfindung. Wir waren bisher eine sehr aktive und forschungsstarke Teilfachhochschule und wollen unsere Vorzüge in diesem Bereich nun noch klarer herausstellen. Es ist für eine kleine Fachhochschule (1900 Studierende und etwa 300 Mitarbeitende) besonders wichtig, innovativ und aktiv zu sein.

Das Schweizer Institut für Informationswissenschaft (SII) ist eines von insgesamt neun Instituten der Hochschule und am Departement für Angewandte Zukunftstechnogien angesiedelt. Es existiert seit nunmehr 23 Jahren und ist inzwischen mit 40 Mitarbeitenden das personal-, umsatz- und forschungsstärkste Institut der Hochschule. Dabei profitierte es in den letzten fünf Jahren von einem starken Wachstum - sowohl die Drittmittel als auch die
Anzahl der Studierenden wie der Mitarbeitenden haben sich in dieser Zeit mehr als verdoppelt. In einer Phase, in der die Informationswissenschaft vor allem im deutschsprachigen Raum von Kürzungen betroffen ist und zunehmend Lehrstühle gestrichen oder nicht neu besetzt werden, agieren wir deutlich und bewusst gegen den Trend. Die Grundlage unseres Erfolgs ist die konsequente Nutzung der Synergien zwischen den klassischen Themen der Informationswissenschaft (z.B. Bibliothek, Information Retrieval) und neuen Inhalten und Methoden (z. B. Datenmanagement, Datenanalyse, User Experience). Die gezielte Auswahl guter Mitarbeiterinnen und Mitarbeiter und die motivierende forschungsfreundliche Struktur im Institut und in der gesamten Hochschule wirkt sich deutlich auch auf die Qualität der Lehre aus.

\section{Forschung}

Die Forschung am SII ist in zwei Forschungsschwerpunkte sowie ein Zentrum aufgeteilt, in denen jeweils zwischen 10 und 15 Wissenschaftlerinnen und Wissenschaftler tätig sind. Der Forschungsschwerpunkt Informationsorganisation besteht aus den Forschungsfeldern Bibliothek und Digitalisierung von Kulturgut, Datenmanagement und Digitale Langzeitarchivierung sowie Bildungsinformatik. Einen weiteren Forschungsschwerpunkt haben wir im Bereich Big Data und Analytics, der sich aus den Forschungsfeldern Data Analytics, Digital Business and Usability Engineering zusammensetzt. Als neueste Erweiterung wurde im September 2019 das Zentrum für Datenanalyse, -visualisierung und -simulation (DAViS) eröffnet, das durch seine Kapazitäten im Hochleistungsrechnen die Forschungsfelder der beiden Forschungsschwerpunkte ergänzt und Kooperationspartnern aus Forschung und Wirtschaft ebenfalls zur Verfügung steht. Auf den nächsten Seiten zeigen wir zahlreiche aktuelle Beispiele unserer Projekte.

*Kontaktperson: Prof. Dr. Ingo Barkow, Fachhochschule Graubünden, Schweizer Institut für Informationswissenschaft, Pulvermühlestrasse 57, 7000 Chur, Schweiz, E-Mail: Ingo.barkow@fhgr.ch 


\section{Lehre}

Die Ergebnisse aus der Forschung fließen unmittelbar in unsere verschiedenen Lehrprogramme ein. Das SII bietet zwei Bachelorstudiengänge, einen Bachelor of Science in Informationswissenschaft sowie einen Bachelor of Science in Digital Business Management, einen Masterstudiengang MSc BA in Information and Data Management sowie einem postgradualen Weiterbildungsstudiengang Certificate of Advanced Studies (CAS) / Master of Advanced Studies (MAS) in Informationswissenschaft an. Das Institut ist permanent engagiert, diese Studiengänge weiterzuentwickeln und durch neue Programme zu ergänzen. Aktuell haben wir beispielsweise umfangreiche Anpassungen in der Studien- und Prüfungsordnung vorgenommen, wobei zahlreiche Module aktualisiert oder geändert wurden. Des Weiteren sind wir im Prozess, den iSchools beizutreten und konzipieren mit Partneruniversitäten ein internationales Doktorandenprogramm.

\section{Fazit}

Unsere Stärke liegt in unserer Fähigkeit zur permanenten Reflexion und Weiterentwicklung. Dadurch sind wir nicht nur für aktuelle Herausforderungen - wie diejenigen, die die laufende Pandemie mit sich bringt - sondern auch für alle weiteren zukünftigen Anforderungen und Entwicklungen gut gerüstet. Nun freuen wir uns, wenn Sie sich bei der Lektüre dieses Heftes selbst ein Bild von uns machen möchten. Ich wünsche Ihnen viel Vergnügen beim Einblick in unsere Projekte und Studienprogramme auf den folgenden Seiten.

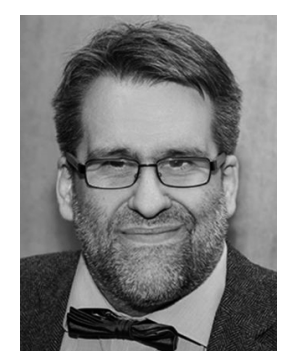

\author{
Prof. Dr. Ingo Barkow \\ Fachhochschule Graubünden \\ Schweizer Institut für \\ Informationswissenschaft \\ Pulvermühlestrasse 57 \\ 7000 Chur \\ Schweiz \\ Ingo.barkow@fhgr.ch
}

Prof. Dr. Ingo Barkow ist seit 2015 an der FH Graubünden beschäftigt, zunächst als Dozent für Datenmanagement, seit 2017 als Professor und seit 2019 als Institutsleiter des Schweizerischen Institut für Informationswissenschaft. Zuvor war er als Data Manager am Deutschen Institut für Internationale Pädagogische Forschung (DIPF) in Frankfurt am Main tätig und hatte dort in den Fachbereichen Forschungsdatenzentrum (FDZ) sowie Zentrum für Technologiebasiertes Assessment (TBA) die technische Leitung inne. 